\title{
Minimum Distance Estimation and Testing of DSGE Models from Structural VARs
}

\author{
PATRICK FÈVE, JULIEN MATHERON
}

AND JEAN-GUILLAUME SAHUC 


\title{
Minimum Distance Estimation and Testing of DSGE Models from Structural VARs*
}

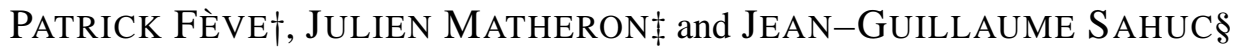 \\ $\dagger$ Toulouse School of Economics (GREMAQ and IDEI) and IUF, Manufacture des Tabacs, Aile J.J. Lafont, bât. F, 21 allée de Brienne, \\ 31000 Toulouse, and Banque de France,DGEI-DEMFI, Paris, France (e-mail: patrick.feve@univ-tlse1.fr) \\ $\ddagger$ Banque de France, DGEI-DEMS-SEPS, 31 rue croix des petits champs, 75049 Paris cedex 1, France (e-mail: julien.matheron@banque- \\ france.fr) \\ §Banque de France, DGEI-DEMS-SEPS, 31 rue croix des petits champs, 75049 Paris cedex 1, and Audencia School of Management, \\ Nantes, France (e-mail: jean-guillaume.sahuc@banque-france.fr)
}

\begin{abstract}
The aim of this paper is to complement the MDE-SVAR approach when the weighting matrix is not optimal. In empirical studies, this choice is motivated by stochastic singularity or collinearity problems associated with the covariance matrix of Impulse Response Functions. Consequently, the asymptotic distribution cannot be used to test the economic model's fit. To circumvent this difficulty, we propose a simple simulation method to construct critical values for the test statistics. An empirical application with US data illustrates the proposed method.
\end{abstract}

\section{Introduction}

The econometrics of Dynamic Stochastic General Equilibrium (DSGE) models has witnessed substantial advances over the recent years. It is nowadays more and more common to take DSGE models to the data using a variety of formal statistical techniques. The present paper is concerned with the estimation and testing principles underlying the popular Minimum Distance Estimation (see Rotemberg and Woodford, 1997, Amato and Laubach, 2003, Christiano, Eichenbaum and Evans, 2005, Giannoni and Woodford, 2005, Altig et al., 2005, Boivin and Giannoni, 2006, Sbordone, 2006, among others).

The Minimum Distance Estimation (MDE) technique consists of estimating the structural parameters of DSGE models so as to minimize a weighted distance between theoretical impulse response functions (IRFs) of key macroeconomic variables to structural shocks and those derived from a Structural Vector Autoregression (SVAR). An attractive feature of the MDE-SVAR approach is that it does not impose a specific stochastic structure of the DSGE model since attention is focused only on those shocks that are relevant for the question under study. This method requires that an auxiliary SVAR model be estimated prior to estimating the DSGE parameters. In doing so,

\footnotetext{
${ }^{*}$ We thank F. Collard, J.P. Florens, C. Bowdler (editor), and an anonymous referee for useful remarks and suggestions. The remaining errors are ours. The views expressed herein are those of the authors and do not necessarily reflect those of the Banque de France.

JEL Classification numbers: C15, C32, E32.
} 
a researcher has access to various types of identifying restrictions which are generally satisfied by broad classes of DSGE models. From this perspective, SVARs constitute useful guides for building empirically plausible models.

The aim of this paper is to provide inference tools complementing the MDE-SVAR framework. We propose a simple simulation technique for testing DSGE models when the weighting matrix is not optimal. As a matter of fact, empirical studies generally do not use the optimal weighting matrix. Instead, researchers resort to a diagonal weighting matrix involving the reciprocal of each IRF variance on the main diagonal. This choice is motivated by practical considerations. Indeed, it is often the case that the covariance matrix of IRFs is not invertible. This can be a direct consequence of a stochastic singularity arising when the number of selected IRFs exceeds the number of estimated parameters in the SVAR. This problem can also result from collinearity problems, which appear when parameter uncertainty in the SVAR is unlikely to significantly affect the covariance matrix of IRFs. In such a case, using a diagonal covariance matrix is a legitimate choice when one seeks to estimate a DSGE model. However, the asymptotic chi-square distribution cannot be used to test the model's fit.

To circumvent this difficulty we propose a simple simulation method that complements the standard MDE-SVAR toolkit. In a first step, the DSGE model is estimated so as to mimic the IRFs from the SVAR. We compute the overidentification test statistic as well as the $t$-statistics. In a second step, we construct simulated versions of these statistics by bootstrapping the SVAR residuals. For each replication, the model's parameters are re-estimated from a centered version of the moment conditions and bootstrap analogs of the test statistics are computed. Replicating this experiment a large number of times, we obtain a population for these statistics, from which it is possible to construct critical values or $P$-values for the relevant tests. The latter can be used to assess the DSGE model in various dimensions.

As a case study, we apply this method to a standard DSGE model with price and wage stickiness. We first estimate with US data a monetary SVAR model and compute the IRFs of output, inflation, wage inflation, and the nominal interest rate to a monetary policy shock. We then estimate and test the DSGE model from these IRFs for two different horizons, one chosen to minimize the Redundant Impulse Response Selection Criterion advocated by Hall et al. (2008) and the other being arbitrarily imposed. In addition, we investigate the sensitivity of our estimation procedure to the choice of weighting matrix. In particular, we adopt the weighting matrix advocated by Christiano et al. (2005), which is a diagonal matrix containing the inverse of each IRF variance along its principal diagonal, as well as an identity matrix, as in Rotemberg and Woodford (1997). Our empirical findings point to the following conclusions: (i) given a weighting matrix, tests of parameter significance are not too sensitive to the chosen IRFs horizon, (ii) given a weighting matrix, the test of overidentification crucially depends on the selected horizon, (iii) all these tests depend importantly on the chosen weighting matrix, and (iv) using the (incorrect) asymptotic distribution often leads to failure to reject the model.

The remainder of the paper is structured as follows. First, we review the MDE-SVAR principle and discuss the bootstrap approach to constructing critical values for test statistics. In a second section, we present an application 
with US data. A last section concludes.

\section{The MDE-SVAR approach}

\section{The minimum distance estimator}

Assume that we seek to estimate an $n_{\psi} \times 1$ vector of structural parameters $\psi$ that characterize a DSGE model. To do so, we focus on an $n_{\theta} \times 1$ vector of empirical moments $\theta$, whose true value is denoted by $\theta_{0}$, and which the economic model is asked to match. In practice, an estimate $\hat{\theta}_{T}$ is substituted for $\theta_{0}$. It is assumed that $\sqrt{T}\left(\hat{\theta}_{T}-\theta_{0}\right) \sim \mathrm{N}\left(0, \Sigma_{\theta}\right)$, where $T$ denotes the sample size. The theoretical counterpart of $\theta$ can be obtained from $\psi$ through the mapping $h(\cdot)$. The Minimum Distance Estimator $\hat{\psi}_{T}$ of $\psi$ is then

$$
\hat{\psi}_{T}=\arg \min _{\psi \in \Psi}\left(h(\psi)-\hat{\theta}_{T}\right)^{\prime} W_{T}\left(h(\psi)-\hat{\theta}_{T}\right) .
$$

Here $\Psi$ is the set of admissible values for the parameters $\psi$ and $W_{T}$ is a definite positive weighting matrix that may depend on the data. Under standard regularity conditions, it can be shown that $\sqrt{T}\left(\hat{\psi}_{T}-\psi_{0}\right) \sim \mathrm{N}\left(0, \Sigma_{\psi}\right)$, where $\psi_{0}$ is $\psi$ 's true value and $\Sigma_{\psi}$ obeys

$$
\Sigma_{\psi}=\left(\frac{\partial h^{\prime}}{\partial \psi} W_{T} \frac{\partial h}{\partial \psi^{\prime}}\right)^{-1} \frac{\partial h^{\prime}}{\partial \psi} W_{T} \Sigma_{\theta} W_{T} \frac{\partial h}{\partial \psi^{\prime}}\left(\frac{\partial h^{\prime}}{\partial \psi} W_{T} \frac{\partial h}{\partial \psi^{\prime}}\right)^{-1}
$$

where the derivatives are evaluated at $\theta_{0}$ and $\psi_{0}$. Let us define $g(\psi, \theta) \equiv h(\psi)-\theta$ and the $J$-statistic $J(\psi) \equiv$ $g(\psi, \theta)^{\prime} W_{T} g(\psi, \theta)$.

When $\Sigma_{\theta}$ is invertible, an "optimal" Minimum Distance Estimator is available when $W_{T} \rightarrow \Sigma_{\theta}^{-1}$. This estimator is optimal in the sense that it delivers the smallest variance for $\psi$ in the considered class of Minimum Distance estimators (see Gourieroux and Monfort, 1995). In this case, it can be shown that $J$ is asymptotically distributed as a $\chi^{2}$ with degrees of freedom equal to $n_{\theta}-n_{\psi}$.

\section{The MDE-SVAR approach and difficulties thereof}

In the MDE-SVAR, the vector of empirical moments $\theta$ is constituted of IRFs drawn from a SVAR. Suppose that we are interested in an $n_{Z} \times 1$ vector of variables $Z_{t}$, the dynamics of which is characterized by the canonical VAR

$$
Z_{t}=A_{1} Z_{t-1}+\cdots+A_{\ell} Z_{t-\ell}+u_{t}, \quad u_{t} \sim i i d(0, \Sigma)
$$

where $\ell \geq 1$. Economic theory can be used to interpret $u_{t}$ as linear combinations of structural shocks $\eta_{t}$, with $\mathrm{E}\left\{\eta_{t} \eta_{t}^{\prime}\right\}=I_{n_{Z}}$. More precisely, there exists a non singular matrix $S$ such that $u_{t}=S \eta_{t}$. Without loss of generality, we consider a single structural shock $s, s \in\left\{1, \ldots, n_{Z}\right\}$. For $k \geq 0$, let us define the vectors $\zeta_{k}=\partial Z_{t+k} / \partial \eta_{t}^{s}$ and $\theta \equiv \operatorname{vec}\left(\left[\zeta_{0}, \zeta_{1}, \ldots, \zeta_{k}\right]^{\prime}\right)$, where the $\operatorname{vec}(\cdot)$ operator stacks the columns of a matrix. 
In order to implement the MDE-SVAR approach, a choice of $k$ must be made. Here, we adopt the Redundant Impulse Response Selection Criterion (RIRSC) advocated by Hall et al. (2008). Formally, the horizon of the IRFs obeys

$$
\hat{k}_{T}=\arg \min _{k \in \mathscr{K}}\left\{\log \left(\operatorname{det}\left(\Sigma_{\psi}(k)\right)\right)+\frac{n_{\theta}(k) \log (T)}{T}\right\},
$$

where $\Sigma_{\psi}(k)$ is the variance-covariance matrix of the estimated parameters $\psi$ and $n_{\theta}(k)$ is the number of elements in the vector of stacked IRFs $\theta$. Notice that our notations make explicit the dependence of both quantities on the selected horizon $k$. Finally, $\mathscr{K} \equiv\left\{k_{\min }, \ldots, k_{\max }\right\}$ is the set of admissible values for $k$. As explained by Hall et al. (2008), imposing the corresponding horizon helps to select the most informative IRFs about the DSGE model parameters.

As explained above, the weighting matrix $W_{T}$ in eq. (1) should be equal to the inverse of the covariance matrix of $\theta$. In practice however, this choice is not always feasible. Indeed, $\theta$ contains, at most, as many free elements as the vector of VAR parameters, $\gamma$. In many empirical applications, $\theta$ is larger than $\gamma$. For example, what turns out to be important for identifying an economic model is the persistence embedded in an IRF shape, which leads to include a large number of moments in $\theta$. In addition, a business cycle student is often interested in the comovement patterns of several aggregate variables in response to a structural shock. This mechanically increases the size of $\theta$. All these applied requirements preclude computing the inverse of $\Sigma_{\theta}$. The lack of invertibility of $\Sigma_{\theta}$ can also arise as a consequence of collinearity problems, which appear when sampling uncertainty is the main source of IRFs variability. This is even more stringent than the previous limitations.

As a consequence, a common and legitimate choice in the applied MDE-SVAR literature is to set $W_{T}$ equal to a matrix containing the inverse of the variances of the elements of $\theta$ along its diagonal and zeros elsewhere (Christiano et al., 2005, Boivin and Giannoni, 2006). Such a diagonal weighting matrix allows us to eschew the stochastic singularity problems discussed above. Another legitimate choice adopted in the literature is to use an identity matrix (Rotemberg and Woodford, 1997, Amato and Laubach, 2003).

However this approach also entails a cost. Since $W_{T}$ is not the optimal weighting matrix, the $J$ statistic is not asymptotically distributed as a $\chi^{2}$ with $n_{\theta}-n_{\psi}$ degrees of freedom. Yet we are interested in testing the model's fit. Thus it is important to know how $J\left(\hat{\psi}_{T}\right)$ is distributed. Here, we propose a simple simulation approach that allows us to compute the critical values of this test statistic.

\section{A bootstrap analog of the MDE-SVAR}

We adapt the methodology advocated by Hall and Horowitz (1996) to the MDE-SVAR and proceed as follows. We start by computing $N$ bootstrap replications of the structural VAR. Let $\left\{u_{t}\right\}_{t=1}^{T}$ denote the canonical VAR residuals. We construct $N$ new time series residuals $\left\{\tilde{u}_{t}^{i}\right\}_{t=1}^{T}, i=1, \ldots, N$, where the $t$ th element of $\left\{\tilde{u}_{t}^{i}\right\}_{t=1}^{T}$ is drawn with replacement from $\left\{u_{t}\right\}_{t=1}^{T}$. Using the estimated VAR coefficients and initial historical conditions, we 
construct $N$ time series of $Z_{t},\left\{\tilde{Z}_{t}^{i}\right\}_{t=1}^{T}$. The canonical residuals being iid, no block-bootstrap methods are needed. The temporal dependence in $Z_{t}$ is captured by the parametric SVAR model.

For each replication, the VAR specified in eq. (2) is estimated and the impulse responses computed using the bootstrap analog of the identifying matrix $S$. The resulting population of stacked IRFs is denoted by $\left\{\tilde{\theta}^{i}\right\}_{i=1}^{N}$ and their covariance matrix by $\Sigma_{\theta}$. Then, $W_{T}$ is the inverse of the matrix containing the diagonal elements of $\Sigma_{\theta}$ along its diagonal and zeros elsewhere. At each bootstrap replication $\theta^{i}$, we estimate $\tilde{\psi}_{T}^{i}$ so as to minimize

$$
\tilde{J}_{T}^{i} \equiv\left[g\left(\psi, \tilde{\theta}^{i}\right)-\hat{\mu}_{T}\right]^{\prime} W_{T}\left[g\left(\psi, \tilde{\theta}^{i}\right)-\hat{\mu}_{T}\right],
$$

where $\hat{\mu}_{T} \equiv g\left(\hat{\psi}_{T}, \theta\right)$ recenters the bootstrap analog of the moment conditions. As explained by Hall and Horowitz (1996), without recentering, the bootstrap would implement a moment condition that does not hold in the bootstrapped sample.

We also compute

$$
\tilde{\Sigma}_{\psi}^{i}=\left(\left(\tilde{D}^{i}\right)^{\prime} W_{T} \tilde{D}^{i}\right)^{-1}\left(\tilde{D}^{i}\right)^{\prime} W_{T} \Sigma_{\theta} W_{T} \tilde{D}^{i}\left(\left(\tilde{D}^{i}\right)^{\prime} W_{T} \tilde{D}^{i}\right)^{-1}
$$

where

$$
\left.\tilde{D}^{i} \equiv \frac{\partial h}{\partial \psi^{\prime}}\right|_{\tilde{\psi}_{T}^{i}, \tilde{\theta}^{i}}
$$

We thus obtain a bootstrap analog of a $t$-test of significance of each component of $\psi$. The associated bootstrapped $t$-statistic is defined by

$$
\tilde{t}_{T r}^{i}=\frac{\tilde{\psi}_{T r}^{i}-\hat{\psi}_{T r}}{\left[\left(\tilde{\Sigma}_{\psi}^{i}\right)_{r, r}\right]^{1 / 2}},
$$

where $\hat{\psi}_{T r}$ is the $r$ th component of $\hat{\psi}_{T}, \tilde{\psi}_{T r}^{i}$ is the $r$ th component of $\tilde{\psi}_{T}^{i}$, and $\left(\tilde{\Sigma}_{\psi}^{i}\right)_{r, r}$ is the $(r, r)$ element of $\tilde{\Sigma}_{\psi}^{i}$. This yields a population of $t$-statistics, $\left\{\tilde{t}_{T r}^{i}\right\}_{i=1}^{N}$, and of $J$ statistics, $\left\{\tilde{J}_{T}^{i}\right\}_{i=1}^{N}$, from which we obtain critical or $P$-values associated with $J$ and $t$ tests.

\section{Empirical illustration with a New Keynesian DSGE model}

\section{Data and SVAR}

We use data from the US Non Farm Business (NFB) sector over the sample period 1960(1)-2002(4). The variables used are the linearly detrended logarithm of per capita GDP, $\hat{y}_{t}$, the growth rate of GDP's implicit price deflator, $\hat{\pi}_{t}$, and the growth rate of nominal hourly compensation, $\hat{\pi}_{t}^{w} \cdot{ }^{1}$ The monetary policy instrument is assumed to be the Fed Funds rate, $\hat{\imath}_{t}$. We also include two "information" variables in the SVAR model. First, the growth rate of

\footnotetext{
${ }^{1}$ The civilian non-institutional population over 16 is used as our measure of population. The data are extracted from the Bureau of Labor Statistics website, except for the Fed Funds rate and M2 which are obtained from the FREDII database.
} 

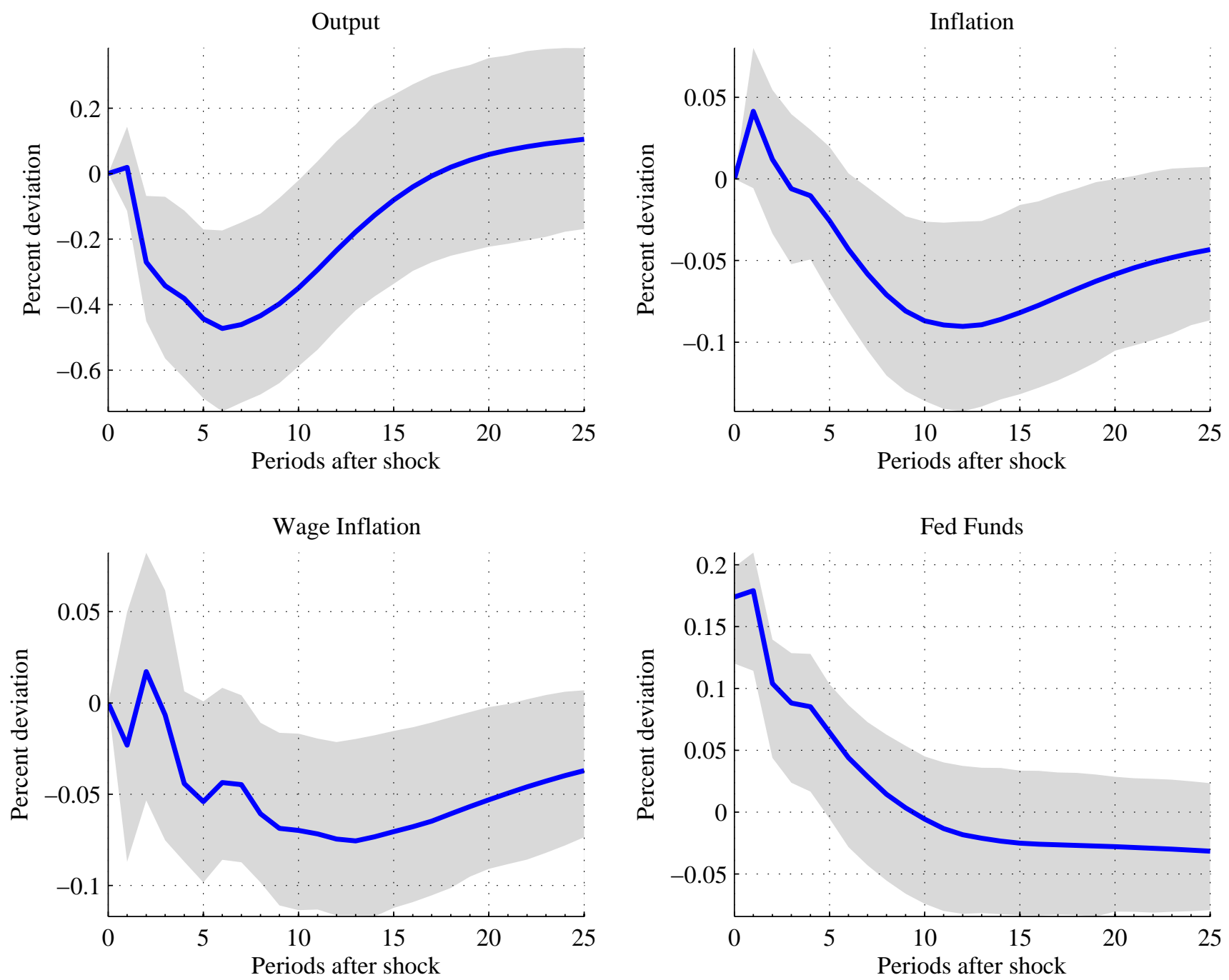

Figure 1. IRFs to a Monetary Policy Shock

the Commodity Research Bureau price index of sensitive commodities, $\hat{\pi}_{t}^{c}$, is included to mitigate the so-called price puzzle. Second, the growth rate of $\mathrm{M} 2, \hat{\xi}_{t}$, is included to exploit information included in money growth.

We set $Z_{t}=\left(\hat{y}_{t}, \hat{\pi}_{t}, \hat{\pi}_{t}^{w}, \hat{\pi}_{t}^{c}, \hat{t}_{t}, \hat{\xi}_{t}\right)^{\prime}$ in eq. (2). Following Christiano et al. (1999), we posit that $S$ is the Cholesky factor of $\Sigma$. Hence, the monetary policy shock is the fifth element of $\eta_{t}$ and $\hat{y}_{t}, \hat{\pi}_{t}, \hat{\pi}_{t}^{w}$, and $\hat{\pi}_{t}^{c}$, by construction, do not respond contemporaneously to such a shock. In addition, the variables of interest are $X_{t}=\left(\hat{y}_{t}, \hat{\pi}_{t}, \hat{\pi}_{t}^{w}, \hat{\imath}_{t}\right)^{\prime}$. The empirical responses of $X_{t}$ are reported on figure 1, with $k=25$. The plain line is our point estimates of the empirical responses of $X_{t}$ and the shaded areas indicate the asymptotic $95 \%$ confidence interval about the point estimates.

Our findings echo previous results reported by Christiano et al. (1999). Output initially responds very little, and then sharply drops, with an inverted hump pattern. The response of inflation displays a persistent U-shaped profile, with a narrow confidence interval. Inflation's lowest response is reached several quarters after output has 
reached its trough. The response of wage inflation is qualitatively similar, with a trough response slightly lagging that of inflation. As discussed in Woodford (2003), the delayed response of inflation is a key stylized fact that any monetary DSGE model should accurately mimic. The Federal Funds rate instantaneously increases, and then gradually declines. These IRFs are the moments used to estimate the DSGE model which we next briefly expound.

\section{A New Keynesian DSGE model}

We consider a standard New Keynesian model with price and wage stickiness along the lines of Giannoni and Woodford (2005) and Galí and Rabanal (2004) which embeds the same timing restrictions as the previous SVAR. To achieve this, we assume that output, inflation, and wage inflation are decided prior to observing the monetary shock.

The first equation is the New Keynesian Phillips curve:

$$
\Delta \hat{\pi}_{t}=\mathrm{E}_{t-1}\left\{\frac{\left(1-\alpha_{p}\right)\left(1-\beta \alpha_{p}\right)}{\alpha_{p}\left(1+\theta_{p} \omega_{p}\right)}\left(\hat{w}_{t}+\omega_{p} \hat{y}_{t}\right)+\beta \Delta \hat{\pi}_{t+1}\right\}
$$

where $\Delta$ denotes the first-difference operator, $\mathrm{E}_{t}$ is the conditional expectation operator, and the variables $\hat{\pi}_{t}, \hat{y}_{t}$, and $\hat{w}_{t}$ are the logdeviations of inflation, output, and real wage, respectively; $\beta \in(0,1)$ is the subjective discount factor, $\alpha_{p} \in[0,1)$ is the degree of nominal rigidity, $\theta_{p}>0$ is the price elasticity of demand, and $\omega_{p}$ is the elasticity of the real marginal cost with respect to the level of production. In the above equation, we implicitly assume that non-reoptimized prices are fully indexed to past inflation, as in Christiano et al. (2005).

A second set of equations defines the IS curve:

$$
\begin{gathered}
\mathrm{E}_{t-1}\left\{\beta b\left(\hat{y}_{t+1}-b \hat{y}_{t}\right)-\left(\hat{y}_{t}-b \hat{y}_{t-1}\right)-(1-\beta b)(1-b) \hat{\lambda}_{t}\right\}=0, \\
\hat{\lambda}_{t}=\hat{\imath}_{t}+\mathrm{E}_{t}\left\{\hat{\lambda}_{t+1}-\hat{\pi}_{t+1}\right\} .
\end{gathered}
$$

where $\hat{\imath}_{t}$, and $\hat{\lambda}_{t}$ are the logdeviations of the gross nominal interest rate, and the representative household's marginal utility of wealth, respectively; $b \in[0,1)$ represents the degree of habit formation.

The wage setting equation is given by:

$$
\hat{\pi}_{t}^{w}-\hat{\pi}_{t-1}=\mathrm{E}_{t-1}\left\{\frac{\left(1-\alpha_{w}\right)\left(1-\beta \alpha_{w}\right)}{\alpha_{w}\left(1+\omega_{w} \theta_{w}\right)}\left(\omega_{w} \phi \hat{y}_{t}-\hat{\lambda}_{t}-\hat{w}_{t}\right)+\beta\left(\hat{\pi}_{t+1}^{w}-\hat{\pi}_{t}\right)\right\}
$$

where $\hat{\pi}_{t}^{w}$ is the logdeviation of wage inflation; $\alpha_{w} \in[0,1)$ is the degree of nominal wage rigidity, $\theta_{w}>0$ is the wage elasticity of labor demand, $\omega_{w}>0$ is the elasticity of the marginal disutility of labor, and $\phi>1$ is the inverse elasticity of output with respect to the labor input. Here, we assume that non-reoptimized wages are fully indexed to past inflation, as in Christiano et al. (2005). Finally, $\hat{\pi}_{t}$ and $\hat{\pi}_{t}^{w}$ are linked together through

$$
\hat{\pi}_{t}^{w}=\hat{w}_{t}-\hat{w}_{t-1}+\hat{\pi}_{t}
$$


The model is closed by postulating the monetary policy rule

$$
\hat{\imath}_{t}=\rho_{i} \hat{\imath}_{t-1}+\left(1-\rho_{i}\right)\left[a_{\pi} \pi_{t}+a_{y} y_{t}\right]+\sigma_{\epsilon} \epsilon_{t}
$$

where $a_{\pi}$ and $a_{y}$ govern the responsiveness of the policy rule to the logdeviations of inflation and output, $\rho_{i}$ is the degree of nominal interest rate smoothing, $\sigma_{\epsilon}>0$, and $\epsilon_{t}$ is an $i i d(0,1)$ monetary policy shock.

\section{Empirical results}

Some parameters are calibrated prior to estimation, either because they can be given values based on great ratios or because they raise specific econometric difficulties (see Canova and Sala, 2006).

First, $\beta=0.99$. As in Christiano et al. (2005) and Altig et al. (2005), $\omega_{w}$ is set to 1 . The value $\phi=1.333$ corresponds to a steady-state share of labor income of $64 \%$, after correcting for the markup. Assuming that the production function is Cobb-Douglas yields $\omega_{p}=\phi-1$. The elasticity of demand for goods $\theta_{p}$ is 11 , as suggested by Basu and Fernald (1997). We set $\theta_{w}$ to 21 as in Christiano et al. (2005). Finally, $a_{\pi}=1.5$ and $a_{y}=0.125$.

We regroup the remaining parameters in $\psi=\left(b, \alpha_{p}, \alpha_{w}, \rho_{i}, \sigma_{\epsilon}\right)^{\prime}$. The latter is estimated using the MDE-SVAR framework laid out above, using different IRF horizons $k$ 's and different weighting matrices $W$. As in the literature, we consider two weighting matrices. The first one corresponds to that containing zeros everywhere except for the principal diagonal which contains the inverse of the IRF's variance, as proposed by Christiano et al. (2005). This will be referred to as the CEE-type weighting matrix. The second one is simply the identity matrix, as in Rotemberg and Woodford (1997). For each choice of weighting matrix, we select the optimal $k$ according to the criterion (3). Notice that since the impact response of the first three variables in $X_{t}$ are degenerate random variables equal to zero, $k=2$ is the minimal horizon for which an overidentification test can be implemented. Indeed, we need at least the IRFs for $t=0,1,2$ to get more than five moments. Alternatively, we impose arbitrarily $k=20$, which corresponds to the horizon that has been considered in the literature, e.g. Christiano et al. (2005).

Figure 2 reports the RIRSC for both weighting matrices. For the CEE-type weighting matrix, we obtain $\hat{k}_{T}=7$, which means that we consider the IRFs of $X_{t}$ from $t=0$ to $t=7$, excluding the first three elements, so that $n_{\theta}=29$. For the identity matrix, we obtain $\hat{k}_{T}=5$, so that in this case $n_{\theta}=21$.

Table 1 reports estimated values for the structural parameters, together with $P$-values obtained from the bootstrapped distribution. The table also reports the $J$ statistic for the test of overidentifying restrictions, together with $P$-values computed from the bootstrapped distribution. In each case, we set $N=1000 .^{2}$

The table suggests the following conclusions. First, given a weighting matrix, the parameters estimates do not depend too much on $k$. With both weighting matrices, we obtain significant parameter estimates either with $\hat{k}_{T}$ or

${ }^{2}$ The corresponding $P$-value is obtained as follows. We first apply a Gaussian kernel with positive support to the simulated statistics $\left\{\tilde{J}_{T}^{i}\right\}_{i=1}^{N}$. The $P$-value is then simply obtained by a piecewise cubic spline interpolation procedure. 

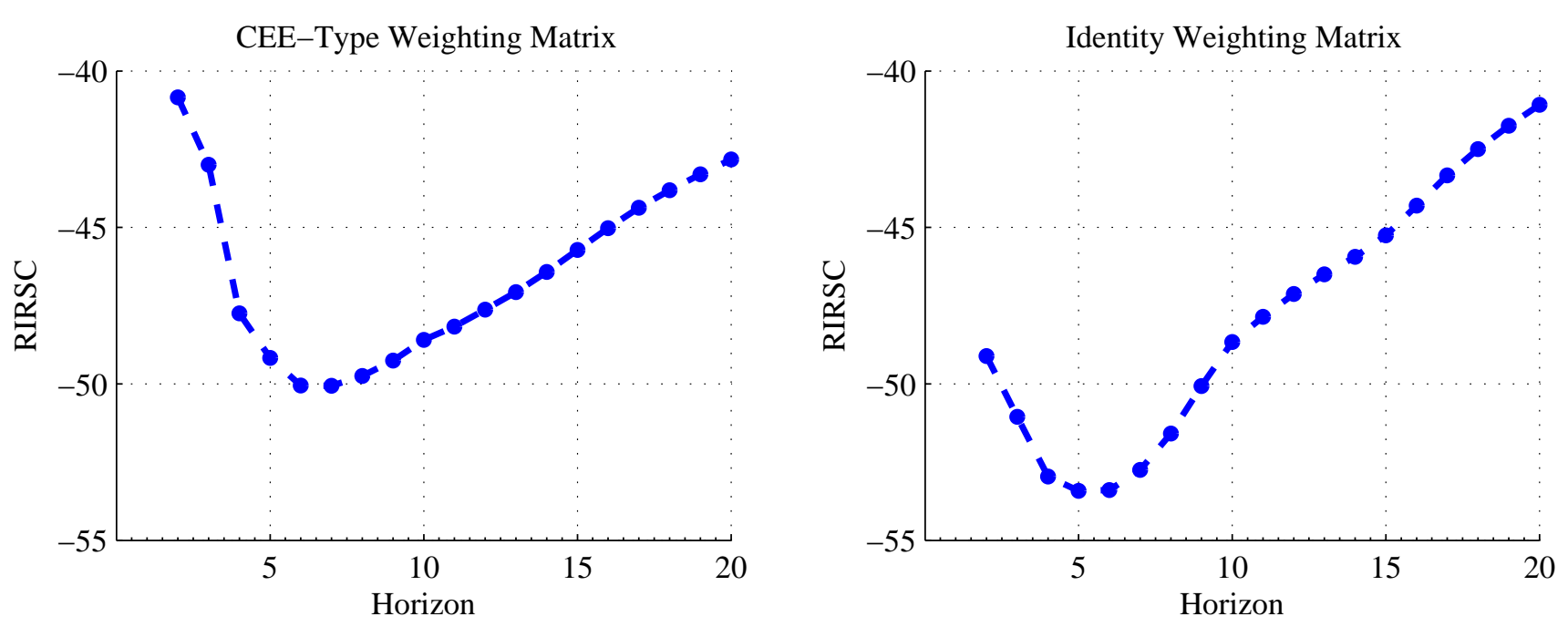

Figure 2. Redundant Impulse Response Selection Criterion

with $k=20$. Second, given a weighting matrix, the $P$-value of the overidentification test crucially depends on the chosen $k$. With either weighting matrices, the model does not pass the identification test when $k$ is set to $\hat{k}_{T}$ while we fail to reject the model when we set $k=20$. Third, when we set $k=20$, our parameter estimates do not depend too much on the weighting matrix and we also reach similar conclusions in terms of model's fit. However, when we adopt the horizon minimizing the RIRSC, the choice of weighting matrix severely impacts on the parameters estimates and the model is rejected in both cases. For each of the four cases considered in our analysis, the results of the overidentification tests are illustrated in figure 3, which reports the cumulative distribution function of the $J$ statistic. Finally, notice that our estimation are in line with previous findings in the literature: the model is characterized by high degrees of habits $(b)$ and nominal rigidities $\left(\alpha_{p}, \alpha_{w}\right)$.

We now propose to assess the importance of carefully deriving a simulated distribution of $J$ statistics as opposed to resorting to an incorrect asymptotic $\chi^{2}$ distribution (e.g., Boivin and Giannoni, 2006). We compare the outcome of the overidentification test when we use the CEE-type weighting matrix. As table 1 suggests, the model is rejected for $\hat{k}_{T}=7$. Using the same horizon but incorrectly resorting to a $\chi^{2}(24)$, we would obtain a $P$-value of $26 \%$. Incorrectly resorting to the asymptotic $\chi^{2}$ distribution would unambiguously lead to non-rejection of the model. When $k=20$, the data are barely supportive of the model, given that the $P$-value is smaller than $10 \%$. However, using the incorrect asymptotic $\chi^{2}(76)$ distribution, we would obtain a $P$-value of $91.3 \%$.

\section{Conclusion}

This paper has proposed a simple simulation method for computing critical or $P$-values of test statistics based on the MDE-SVAR approach. The method is especially suitable when the weighting matrix is not optimal. In empirical studies, this choice is essentially motivated by stochastic singularity and collinearity problems that preclude 
TABLE 1

Estimation Results

\begin{tabular}{|c|c|c|c|c|}
\hline & \multicolumn{4}{|c|}{ Selected Horizon } \\
\hline & \multicolumn{2}{|c|}{ CEE-Type } & \multicolumn{2}{|c|}{ Identity } \\
\hline & $\hat{k}_{T}=7$ & $k=20$ & $\hat{k}_{T}=5$ & $k=20$ \\
\hline \multicolumn{5}{|c|}{ Parameters } \\
\hline \multirow[t]{2}{*}{$b$} & 0.809 & 0.860 & 0.917 & 0.846 \\
\hline & {$[0.000]$} & {$[0.000]$} & {$[0.000]$} & {$[0.000]$} \\
\hline \multirow[t]{2}{*}{$\alpha_{p}$} & 0.842 & 0.811 & 0.933 & 0.803 \\
\hline & {$[0.000]$} & {$[0.000]$} & {$[0.000]$} & {$[0.100]$} \\
\hline \multirow[t]{2}{*}{$\alpha_{w}$} & 0.824 & 0.855 & 0.913 & 0.830 \\
\hline & {$[0.599]$} & {$[0.300]$} & {$[0.000]$} & {$[8.791]$} \\
\hline \multirow[t]{2}{*}{$\rho_{i}$} & 0.925 & 0.952 & 0.970 & 0.955 \\
\hline & {$[0.000]$} & {$[0.000]$} & {$[0.000]$} & {$[0.000]$} \\
\hline \multirow[t]{2}{*}{$\sigma_{\nu}$} & 0.157 & 0.139 & 0.132 & 0.129 \\
\hline & {$[0.000]$} & {$[0.000]$} & {$[0.000]$} & {$[0.000]$} \\
\hline \multicolumn{5}{|c|}{ Overidentification Test } \\
\hline \multirow[t]{2}{*}{$J\left(\hat{\psi}_{T}\right)$} & 27.995 & 59.876 & 36015.973 & 80192.180 \\
\hline & {$[1.322]$} & {$[6.668]$} & {$[0.588]$} & {$[16.252]$} \\
\hline
\end{tabular}

Notes: The $P$-values, in brackets, are in percentage. In the "Parameter" panel, the null hypothesis being tested is that the corresponding parameter is zero. In the "Overidentification Test" panel, the null hypothesis being tested is that the $J$ statistic is zero.

using the covariance matrix of IRFs. A diagonal weighting matrix allows a researcher to solve the invertibility problem, but the asymptotic distribution of the MDE-SVAR for the test of overidentifying restrictions can no longer be used. Consequently, the DSGE model's fit cannot be properly evaluated. The use of bootstrap methods, still maintaining diagonal weighting matrix, gives us the opportunity to conduct statistical inference. Bootstrap simulations of the SVAR models and repeated estimation of the model using MDE allows us to compute critical values for test statistics and to conduct a proper evaluation of DSGE models. We illustrate the potential usefulness of the proposed approach with US data. 

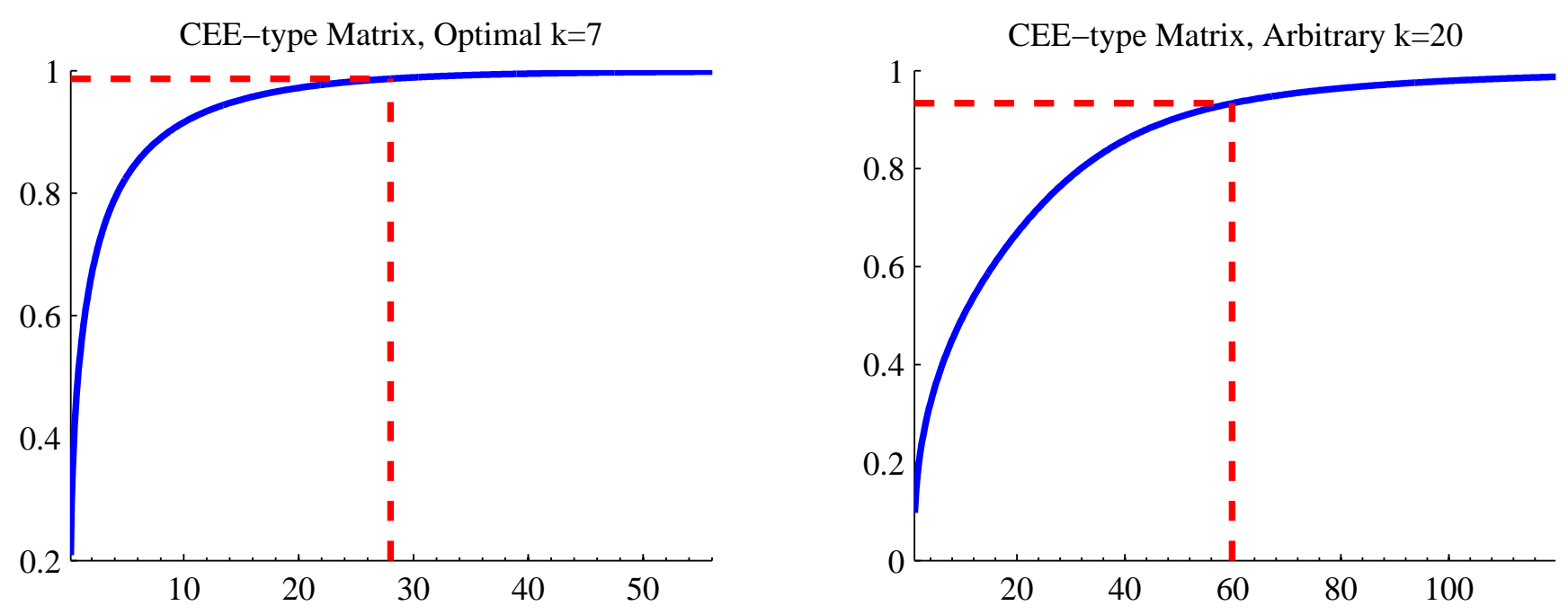

Identity Matrix, Optimal k=5
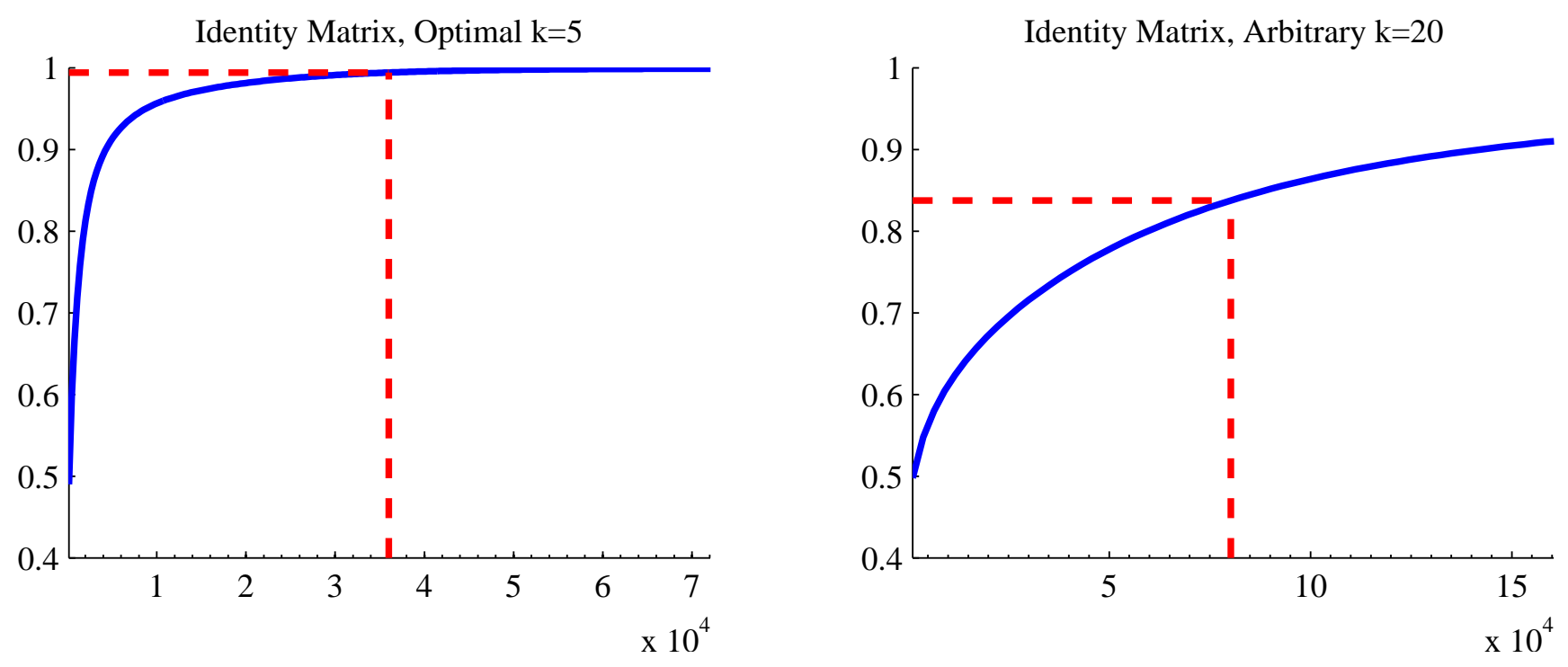

Figure 3. Bootstrapped CDF of $J$. The vertical line corresponds to the estimated $J$ statistic.

\section{References}

Altig, D., Christiano, L.J., Eichenbaum, M. and Lindé, J. (2005). 'Firm-specific capital, nominal rigidities and the business cycle', Working Paper No. 11034, NBER.

Amato, J. and Laubach, T. (2003). 'Estimation and control of an optimization-based model with sticky prices and wages', Journal of Economic Dynamics and Control, Vol. 27, pp. 1181-1215.

Basu, S. and Fernald, J.G. (1997). 'Returns to scale in U.S. production: Estimates and implications', Journal of Political Economy, Vol. 105, pp. 249-283.

Boivin, J. and Giannoni, M. (2006). 'Has monetary policy become more effective', Review of Economics and Statistics, Vol. 88, pp. 445-462.

Canova, F. and Sala, L. (2006). 'Back to the square one: Identification issues in DSGE models', mimeo UPF. 
Christiano, L.J., Eichenbaum, M. and Evans, C.L. (1999). 'Monetary policy shocks: What have we learned and to what end?', in J.B. Taylor and M. Woodford (eds.), Handbook of Macroeconomics, Vol. 1A. Elsevier, Amsterdam, pp. 65-148.

Christiano, L.J., Eichenbaum, M. and Evans, C. (2005). 'Nominal rigidities and the dynamic effects of a shock to monetary policy', Journal of Political Economy, Vol. 113, pp. 1-45.

Galí, J. and Rabanal, P. (2004). 'Technology shocks and aggregate fluctuations: how well does the RBC model fit Postwar U.S. data?' in M. Gertler and K. Rogoff (eds.), NBER Macroeconomics Annual, pp. 225-288.

Giannoni, M. and Woodford, M. (2005). 'Optimal inflation targeting rules', in B.S. Bernanke and M. Woodford (eds.), Inflation Targeting, University of Chicago Press.

Gourieroux, C. and Monfort, A. (1995). Statistics and Econometric Models, Cambridge University Press.

Hall, A., Inoue, A., Nason, J.M. and Rossi, B. (2008). 'Information criteria for impulse response function matching estimation of DSGE models'. Working Paper No. 2007-10a, Federal Reserve Bank of Atlanta.

Hall, P. and Horowitz, J. (1996). 'Bootstrap critical values for tests based on generalized-method-of-moments estimators', Econometrica, Vol. 64, pp. 891-916.

Rotemberg, J.J. and Woodford, M. (1997). 'An optimization-based econometric framework for the evaluation of monetary policy', in B.S. Bernanke and J.J. Rotemberg (eds.), NBER Macroeconomics Annual, MIT Press, Cambridge, pp. 297-346.

Sbordone, A.M. (2006). 'U.S. wage and price dynamics: A limited-information approach', International Journal of Central Banking, Vol. 2, pp. 155-191.

Woodford, M. (2003). Interest and Prices: Foundation of a Theory of Monetary Policy. Princeton University Press, Princeton. 